\title{
Línea Base Radiológica Ambiental en el Centro Nuclear RACSO (1980-2020)
}

\author{
JM. Osores, J. Martínez, D. Huarachi, R. Koga, L. Huaman, R. Jara \\ Instituto Peruano de Energía Nuclear, Av. Canada 1480, Lima, Perú \\ Enviado el 16 de noviembre del 2021. Aceptado el 8 de enero del 2022
}

DOI: https://doi.org/10.33017/RevECIPeru2021.0006/

\begin{abstract}
Resumen
Se realizó la evaluación de los niveles de radiactividad comprendida entre los años 1980 y 2020 con la finalidad de establecer una línea base radiológica ambiental del área de influencia del Centro Nuclear Oscar Miró Quesada de la Guerra "Racso". Durante este periodo, se determinó una tasa equivalente de dosis ambiental comprendida entre 0,07 y 0,14 microSievert/hora, se verificó la presencia continua de descargas atmosféricas de l-131 como parte del proceso de producción de este radioisótopo para aplicaciones médicas. Además fueron identificados otros radionúclidos antropogénicos como Na-22, Mn-54, Co-60, Nb-95, Zr-95, Zn-65, Te-123m, Tc-99m, Cs-134 y Cs-137, distribuidos en diferentes matrices ambientales como aire, agua, lodo residual, suelo y bioindicadores. Sin embargo, todos los niveles de concentración de actividad no representan riesgo radiológico para la población y el ambiente.
\end{abstract}

Descriptores: radiactividad ambiental, línea base, vigilancia radiológica

\section{Abstract}

The evaluation of radioactivity levels between 1980 and 2020 was carried out in order to establish an environmental radiological baseline of the area of influence of the Oscar Miró Quesada de la Guerra "Racso" Nuclear Center. During this period, an equivalent rate of environmental dose between 0.07 and 0.14 microSievert / hour was determined, the continuous presence of atmospheric discharges of I-131 was verified as part of the production process of this radioisotope for medical applications. In addition, other anthropogenic radionuclides were identified such as Na-22, Mn-54, Co-60, Nb-95, Zr-95, Zn-65, Te-123m, Tc-99m, Cs-134 and Cs-137, distributed in different environmental matrices such as air, water, residual sludge, soil and bioindicators. However, all levels of activity concentration do not represent a radiological risk for the population and the environment.

Keywords: environmental radioactivity, baseline, radiological monitoring 


\section{Introducción}

El Organismo Internacional de Energía Atómica (OIEA) establece que el objetivo de seguridad en la evaluación del emplazamiento de las instalaciones nucleares consiste en caracterizar los peligros externos naturales e inducidos por el hombre que podrían afectar la seguridad de la instalación nuclear, a fin de proporcionar información adecuada para demostrar la protección de las personas y el medio ambiente frente a los efectos nocivos de las radiaciones ionizantes por lo que recomienda llevar a cabo una revisión independiente de los parámetros de diseño específicos del emplazamiento, y de la evaluación del impacto radiológico potencial de la instalación nuclear sobre las personas y el medio ambiente [1].

Los estados miembros del OIEA llevan a cabo la vigilancia de la radiactividad ambiental con fines de verificación en las instalaciones nucleares con el objetivo evaluar la fluctuación a corto y largo plazo de los elementos radiactivos detectados por encima de la concentración mínima detectable (MDC) y sus orígenes utilizando relaciones de concentración entre estos radionúclidos [2].

En el Perú, el Centro Nuclear Oscar Miró Quesada de la Guerra "Racso" del Instituto Peruano de Energía Nuclear (IPEN), cuenta con instalaciones y laboratorios que procesan y/o manipulan materiales radiactivos con múltiples propósitos [3]. Luego del inicio de las operaciones del reactor nuclear RP-10

\section{Metodología}

Se realizó la recolección de toda información relacionada sobre los niveles de radiactividad registrados en el área de influencia del Centro Nuclear Oscar Miró Quesada de la Guerra "Racso". Las fuentes de información que fueron consideradas son las publicaciones en revistas nacionales y la base de datos disponible en los archivos del Laboratorio de Radioecología del IPEN. Esta información comprende registros desde el año 1980 a la actualidad.

Se establecieron 12 Estaciones de Monitorización Radiológica Ambiental (EMRA) las cuales se detallan en la Figura 1 y la Tabla 1.

Las lecturas del equivalente de dosis se llevaron a cabo con detectores termoluminiscentes ambientales (TLD) y la tasa de dosis se calculó en función del tiempo de monitorización. en 1989, se tuvo la necesidad de organizar un adecuado registro de los niveles de radiactividad en su área de influencia ambiental, previa al funcionamiento y durante la etapa operacional de esta instalación [4].

En el 2019, IPEN priorizó el fortalecimiento de las actividades de los laboratorios de especializados en la prestación de servicios tecnológicos orientados a radioecología, dosimetría biológica y mediciones de radón en donde una de las medidas de control constituye el establecimiento de la línea base radiológica ambiental en torno al área de influencia del centro nuclear.

El objetivo de esta línea base consiste en la elaboración de una base de datos que sirva como herramienta para controlar los niveles de descarga de las instalaciones nucleares y radiactivas del centro nuclear mediante el contraste de los resultados del Programa de Vigilancia Radiológica Ambiental en ejecución con los valores referenciales de los niveles de radiactividad en el entorno.

Se presenta la línea base radiológica ambiental correspondiente al periodo 1980-2020, que incluye la recopilación, tabulación y evaluación de los resultados de radiactividad desde el inicio de los estudios pre-operacionales en el área de influencia del Centro Nuclear Oscar Miró Quesada de la Guerra "Racso"

El monitoreo de I-131 en aire se llevó a cabo con cartuchos de carbón activado acoplados a un muestreador de aire y el análisis se realizó en un sistema de espectrometría gamma de centelleo sólido de yoduro de sodio $\mathrm{Nal}(\mathrm{TI})$. El análisis de las otras muestras ambientales se llevó a cabo en un sistema de espectrometría gamma de alta resolución de germanio hiperpuro [5].

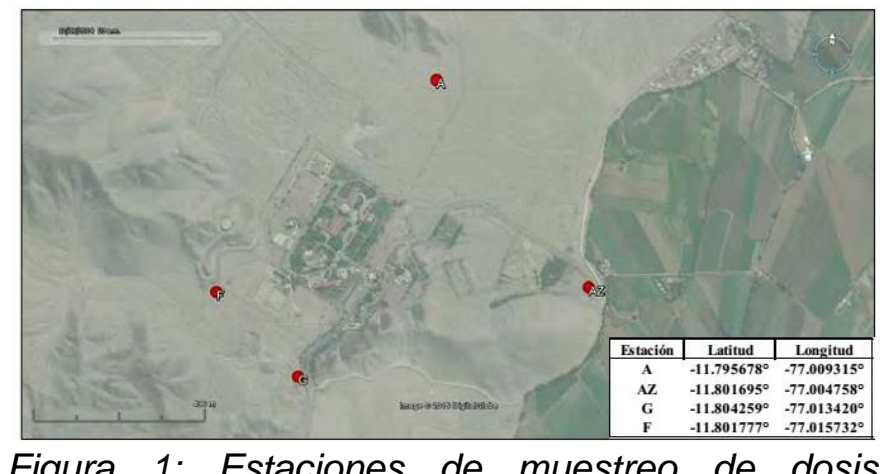

Figura 1: Estaciones de muestreo de dosis ambiental 
Tabla 1: Detalles geográficos referenciales de las EMRA

\begin{tabular}{|l|c|c|c|}
\hline \multirow{2}{*}{ Nombre del lugar } & \multicolumn{3}{|c|}{ Coordenadas Geográficas } \\
\cline { 2 - 4 } & Latitud & Longitud & $\begin{array}{c}\text { Altitud } \\
\text { (msnm) }\end{array}$ \\
\hline $\begin{array}{l}\text { Edificio de Seguridad } \\
\text { Radiológica (SERV) }\end{array}$ & $-11,8015^{\circ}$ & $-77,0123^{\circ}$ & 411 \\
\hline $\begin{array}{l}\text { Jardines posteriores } \\
\text { PPR }\end{array}$ & $-11,7994^{\circ}$ & $-77,0125^{\circ}$ & 419 \\
\hline $\begin{array}{l}\text { Jardines posteriores } \\
\text { RP10 }\end{array}$ & $-11,7985^{\circ}$ & $-77,0117$ & 419 \\
\hline $\begin{array}{l}\text { Planta de tratamiento } \\
\text { efluentes cloacales }\end{array}$ & $-11,8013^{\circ}$ & $-77,0064^{\circ}$ & 385 \\
\hline $\begin{array}{l}\text { Pozo de agua } \\
\text { subterránea }\end{array}$ & $-11,5025^{\circ}$ & $-77,0033^{\circ}$ & 360 \\
\hline $\begin{array}{l}\text { Centro poblado } \\
\text { Huarangal }\end{array}$ & $-11,7951^{\circ}$ & $-77,0005^{\circ}$ & 385 \\
\hline $\begin{array}{l}\text { Río Chillón } \\
\text { Área posterior del } \\
\text { edificio SERV }\end{array}$ & $-11,8055^{\circ}$ & $-76,9971^{\circ}$ & 353 \\
\hline $\begin{array}{l}\text { Perimetro del Centro } \\
\text { Nuclear }\end{array}$ & $-11,7957^{\circ}$ & $-77,0093^{\circ}$ & 415 \\
\hline $\begin{array}{l}\text { Perimetro del Centro } \\
\text { Nuclear }\end{array}$ & $-11,8017^{\circ}$ & $-77,0048^{\circ}$ & 381 \\
\hline $\begin{array}{l}\text { Perimetro del Centro } \\
\text { Nuclear }\end{array}$ & $-11,8043^{\circ}$ & $-77,0134^{\circ}$ & 411 \\
\hline $\begin{array}{l}\text { Perimetro del Centro } \\
\text { Nuclear }\end{array}$ & $-11,8018^{\circ}$ & $-77,0157^{\circ}$ & 433 \\
\hline
\end{tabular}

Los registros de radiactividad ambiental fueron agrupados en función a los años de monitoreo y se estableció el rango y la mediana para cada uno de los parámetros evaluados.

\section{Resultados y Discusión de resultados}

La Tabla 2 y la Figura 2 presentan los valores y la frecuencia de la tasa de equivalente de dosis ambiental desde al año 1980 cuyas primeras lecturas fueron realizadas por miembros del programa de cooperación con la Comisión de Energía Atómica de Argentina [6].

Los valores se han mantenido estables durante el periodo de tiempo de evaluación con un rango comprendido entre 0,07 y 0,14 microSievert/hora.

Las lecturas de esta variable estuvieron a cargo del Laboratorio Secundario de Calibraciones Dosimétricas del IPEN hasta el año 2015, fecha en que el sistema de lectura de los dosímetros ambientales dejó de operar.

Las concentraciones de I-131 en aire presentan una amplia variabilidad en los valores obtenidos dependiendo del año de operación, estos valores tienen un elevado rango de amplitud que va desde los 0,001 a los $13,68 \mathrm{~Bq} / \mathrm{m}^{3}$ con una mediana de $0,65 \mathrm{~Bq} / \mathrm{m}^{3}$ (Figura 3). A la fecha no se han reportado la presencia de otros radionúclidos de origen antropogénico en aire.
Tabla 2: Tasa de Equivalente de Dosis Ambiental $(1980-2015)$

\begin{tabular}{|c|c|c|c|}
\hline Año & $\begin{array}{c}\text { Dosis } \\
\text { (uSv/h) }\end{array}$ & Año & $\begin{array}{c}\text { Dosis } \\
\text { (uSv/h) }\end{array}$ \\
\hline 1980 & 0.1164 & 2003 & 0,1090 \\
\hline 1980 & 0.1210 & 2003 & 0,0830 \\
\hline 1980 & 0.1347 & 2004 & 0,0890 \\
\hline 1980 & 0.1450 & 2004 & 0,0939 \\
\hline 1981 & 0.1059 & 2004 & 0,1040 \\
\hline 1981 & 0.1024 & 2004 & 0,0820 \\
\hline 1981 & 0.1164 & 2013 & 0,0776 \\
\hline 1981 & 0.1279 & 2013 & 0,0731 \\
\hline 2001 & 0.0890 & 2013 & 0,0982 \\
\hline 2001 & 0.0840 & 2013 & 0,0753 \\
\hline 2001 & 0.1070 & 2014 & 0,0845 \\
\hline 2001 & 0.0850 & 2014 & 0,0788 \\
\hline 2002 & 0.0920 & 2014 & 0,1005 \\
\hline 2002 & 0.0830 & 2014 & 0,0776 \\
\hline 2002 & 0.1153 & 2015 & 0,0873 \\
\hline 2002 & 0.0830 & 2015 & 0,0873 \\
\hline 2003 & 0.0900 & 2015 & 0,1010 \\
\hline 2003 & 0.0870 & 2015 & 0,0822 \\
\hline Rango: & $\mathbf{0 , 0 7}-\mathbf{0 , 1 4}$ & Mediana: & $\mathbf{0 , 0 9}$ \\
\hline & & &
\end{tabular}

Figura 2: Frecuencia de equivalente de dosis ambiental

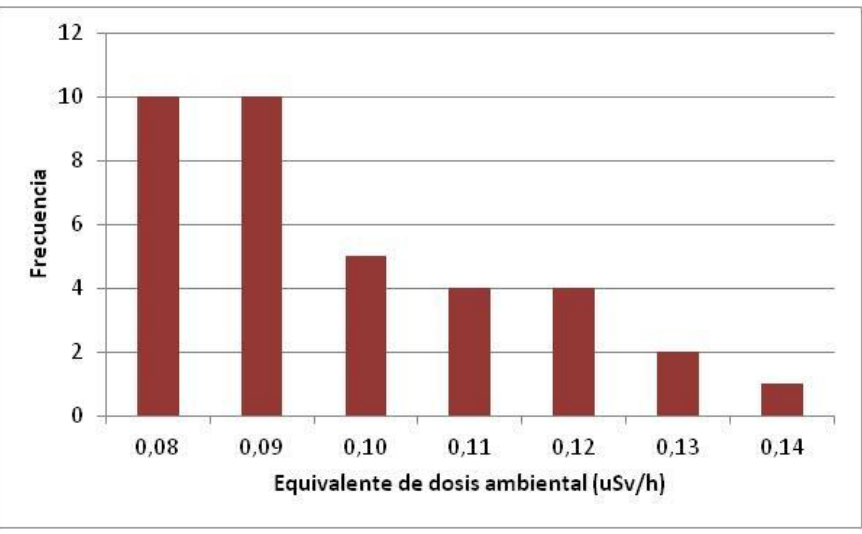

La Comisión Internacional de Protección Radiológica, recomienda el uso de bioindicadores de referencia específicos para la evaluación del impacto en la biota debido a las descargas radiactivas en el ambiente [7], razón por la cual dentro de este periodo de monitoreo se realiza la evaluación del depósito de I-131 descargado al ambiente sobre Cynodon dactylon (Figura 3). 


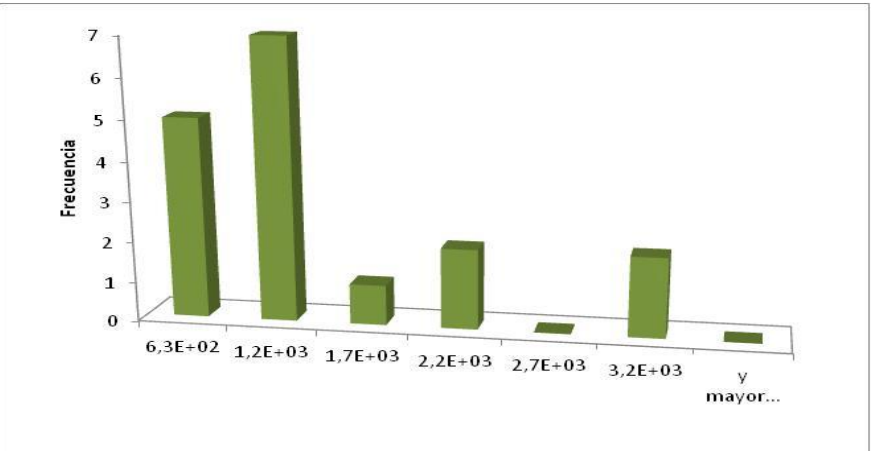

Figura 3: Frecuencia de actividad de $\mid-131$ depositado sobre Cynodon dactylon $\left(\mathrm{Bq} / \mathrm{m}^{2}\right)$

Además se ha encontrado la presencia de diversos radionúclidos de origen antropogénico que son liberados por las instalaciones y laboratorios del centro nuclear a través del sistema de drenaje, el cual deriva hasta la Planta de Tratamiento de Efluentes Cloacales (PTEC) para su disposición final. Este material radiactivo tiende a concentrarse en los lodos cloacales los cuales al ser caracterizados por espectrometría gamma se encontraron concentraciones variables de Co-60, Cs-134, Cs-137, Te-123m, y Mn-54 (Figuras 4, 5, 6, 7 y 8 ) y algunos de manera ocasional (Tabla 3).

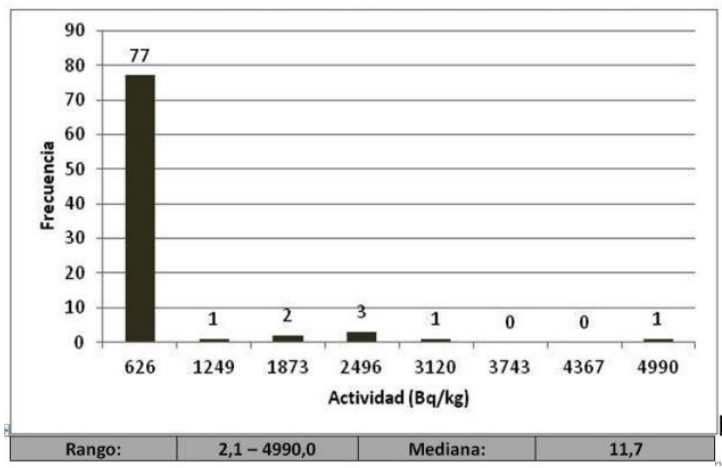

Figura 4: Concentración de actividad de Co-60 en lodo residual

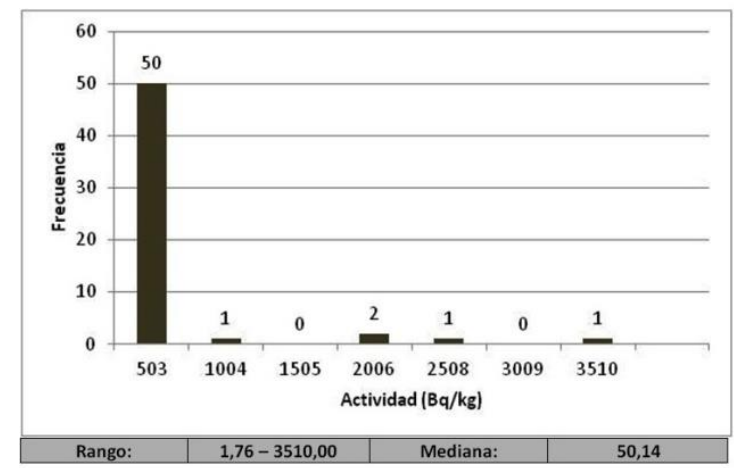

Figura 5: Concentración de actividad de Cs-134 en lodo residual

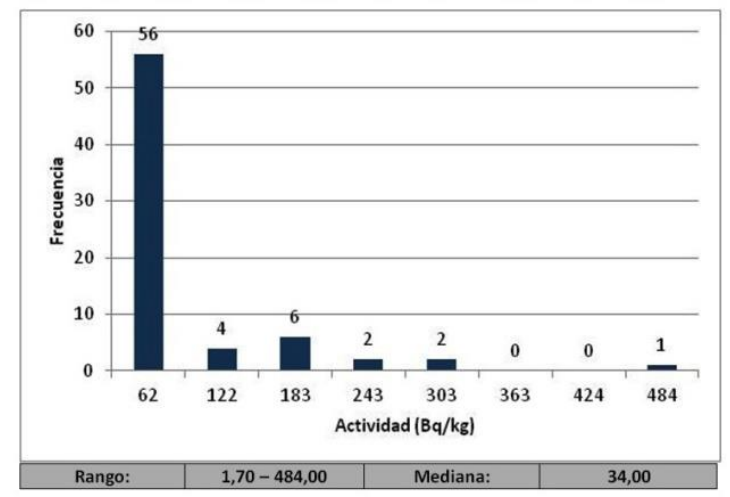

Figura 6: Concentración de actividad de Cs-137 en lodo residual

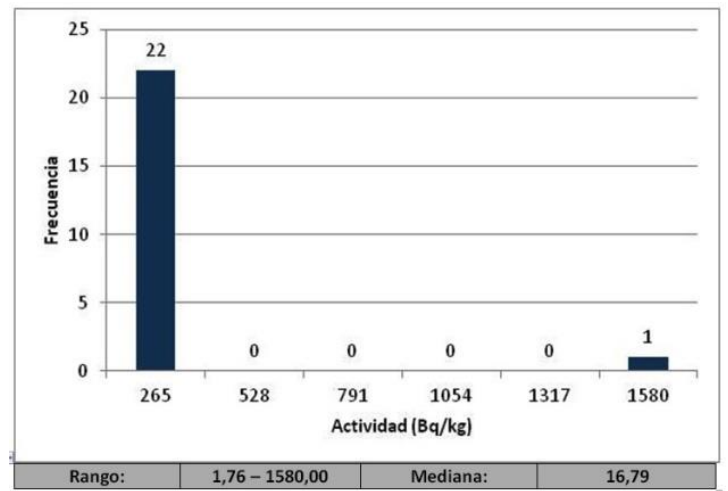

Figura 7: Concentración de actividad de Te-123m en lodo residual

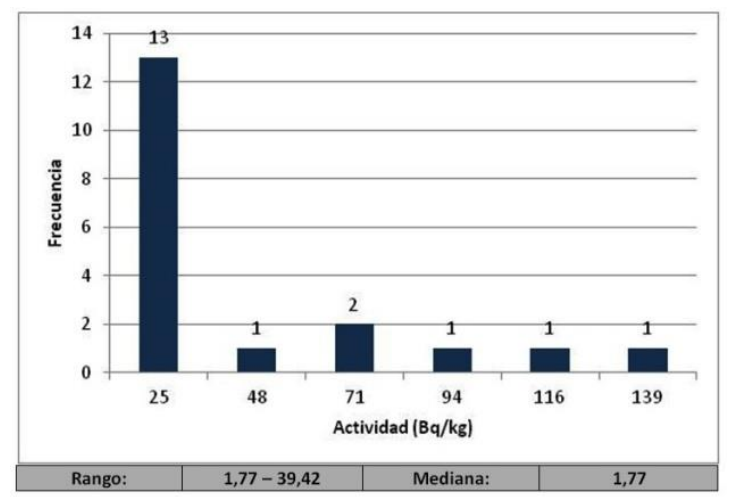

Figura 8: Concentración de actividad de Mn-54 en lodo residual

Tabla 3: Concentración de actividad de diversos radionúclidos ocasionales en lodo residual (2020)

\begin{tabular}{|c|c|c|c|}
\hline $\begin{array}{c}\text { Radio } \\
\text { núclido }\end{array}$ & $\begin{array}{c}\text { Fecha } \\
\text { Referencia }\end{array}$ & $\begin{array}{c}\text { Actividad } \\
\text { (Bq/kg) }\end{array}$ & $\begin{array}{c}\text { Incertidumbre } \\
\text { (Bq/kg) }\end{array}$ \\
\hline Na-22 & $2020-11-30$ & 5,04 & 0,91 \\
\hline $\mathrm{Na}-22$ & $2020-12-09$ & 3,98 & 0,68 \\
\hline Co-57 & $2020-01-14$ & 14,82 & 1,00 \\
\hline $\mathrm{Nb}-95$ & $2020-01-14$ & 12,74 & 3,09 \\
\hline Zn-65 & $2020-01-14$ & 36,37 & 7,83 \\
\hline Zr-95 & $2020-01-14$ & 29,71 & 7,21 \\
\hline
\end{tabular}


Considerando que el lodo residual es retenido en pozas de decaimiento y no llega a otros componentes ambientales, el manejo de estos niveles de contaminación radiactiva no representan riesgo para la biota ni el público del valle cercano a la instalación.

Por otro lado, ninguno de estos niveles representa riesgo para la aparición de efectos determinísticos en las especies biológicas de la zona.
En base a todos los registros evaluados y en función al periodo de monitoreo, los niveles de línea base de radiactividad ambiental en el área de influencia del Centro Nuclear Oscar Miró Quesada de la Guerra se presentan en la Tabla 4.

Tabla 4: Línea base de radiactividad ambiental en el área de influencia del Centro Nuclear RACSO

\begin{tabular}{|c|c|c|c|c|c|}
\hline $\begin{array}{c}\text { Componente } \\
\text { Ambiental }\end{array}$ & Analito & Periodo & Mediana & Rango & Unidad \\
\hline \multirow{4}{*}{ Aire } & Tasa de dosis & $1980-2015$ & 0,09 & $0,07-0,14$ & $\mu \mathrm{Sv} / \mathrm{h}$ \\
\hline & Co-60 & $1992-2020$ & $<0,058$ & ---------- & $\mathrm{Bq} / \mathrm{m}^{3}$ \\
\hline & $\mathrm{I}-131$ & $1992-2020$ & 0,650 & $0,001-13,68$ & $\mathrm{~Bq} / \mathrm{m}^{3}$ \\
\hline & Cs-137 & 2020 & $<0,085$ & ---------- & $\mathrm{Bq} / \mathrm{m}^{3}$ \\
\hline \multirow{6}{*}{ Agua residual } & $\mathrm{K}-40$ & 2020 & 15,73 & $13,74-19,90$ & $\mathrm{Bg} / \mathrm{L}$ \\
\hline & Co-60 & $1999-2020$ & 2,60 & $0,27-6,20$ & $\mathrm{~Bq} / \mathrm{L}$ \\
\hline & Tc-99m & 2020 & 808,61 & $20,21-1597,00$ & $\mathrm{~Bq} / \mathrm{L}$ \\
\hline & $\mathrm{I}-131$ & $2019-2020$ & 2,79 & $0,34-14,72$ & $\mathrm{~Bq} / \mathrm{L}$ \\
\hline & Cs-134 & 2020 & $<0,853$ & --------- & $\mathrm{Bq} / \mathrm{L}$ \\
\hline & Cs-137 & 1996- 2020 & 0,42 & $0,04-3,71$ & $\mathrm{~Bq} / \mathrm{L}$ \\
\hline \multirow{13}{*}{ Lodo residual } & $\mathrm{Na}-22$ & 2020 & 4,51 & $3,98-5,04$ & $\mathrm{Bg} / \mathrm{kg}$ \\
\hline & $\mathrm{K}-40$ & 2020 & 167,94 & $131,27-190,45$ & $\mathrm{~Bq} / \mathrm{kg}$ \\
\hline & $\mathrm{Mn}-54$ & $1994-1999$ & 1,77 & $1,77-39,42$ & $\mathrm{~Bq} / \mathrm{kg}$ \\
\hline & Co-57 & 2020 & 14,82 & $13,82-15,82$ & $\mathrm{~Bq} / \mathrm{kg}$ \\
\hline & Co-60 & 1994- 2020 & 11,70 & $2,10-4990,00$ & $\mathrm{~Bq} / \mathrm{kg}$ \\
\hline & $\mathrm{Nb}-95$ & 2020 & 12,74 & $9,65-15,83$ & $\mathrm{Bg} / \mathrm{kg}$ \\
\hline & Zn-65 & 2020 & 36,37 & $28,54-44,20$ & $\mathrm{Bg} / \mathrm{kg}$ \\
\hline & Zr-95 & 2020 & 29,71 & $22,50-36,92$ & $\mathrm{Bg} / \mathrm{kg}$ \\
\hline & Tc-99m & 2020 & 11555,48 & $127,92-275805$ & $\mathrm{~Bq} / \mathrm{kg}$ \\
\hline & Te-123m & $2016-2020$ & 16,79 & $1,76-1580,00$ & $\mathrm{Bg} / \mathrm{kg}$ \\
\hline & $\mathrm{I}-131$ & 2020 & 5,84 & $2,77-78,22$ & $\mathrm{~Bq} / \mathrm{kg}$ \\
\hline & Cs-134 & $1999-2020$ & 50,14 & $1,76-3510,00$ & $\mathrm{~Bq} / \mathrm{kg}$ \\
\hline & Cs-137 & $1999-2020$ & 34,00 & $1,70-484,00$ & $\mathrm{Bg} / \mathrm{kg}$ \\
\hline \multirow{4}{*}{ Bioindicadores } & Co-60 & 2020 & $<72,74$ & ---------- & $\mathrm{Bq} / \mathrm{m}^{2}$ \\
\hline & Te-123m & 2020 & $<60,68$ & --------- & $\mathrm{Bq} / \mathrm{m}^{2}$ \\
\hline & $\mathrm{I}-131$ & 2020 & 950,20 & $111,01-3235,35$ & $\mathrm{~Bq} / \mathrm{m}^{2}$ \\
\hline & Cs-137 & 2020 & $<43,71$ & --------- & $\mathrm{Bq} / \mathrm{m}^{2}$ \\
\hline \multirow{4}{*}{ Suelo superficial } & $\mathrm{K}-40$ & 2020 & 727,04 & $703,56-750,51$ & $\mathrm{~Bq} / \mathrm{kg}$ \\
\hline & $\mathrm{I}-131$ & 2020 & 5,73 & $4,16-7,30$ & $\mathrm{~Bq} / \mathrm{kg}$ \\
\hline & Cs-137 & 2020 & 2,41 & $2,10-2,71$ & $\mathrm{~Bq} / \mathrm{kg}$ \\
\hline & Cs-137 & 2020 & 2,41 & $2,10-2,71$ & $\mathrm{Bg} / \mathrm{kg}$ \\
\hline \multirow{4}{*}{ Agua de consumo } & $\mathrm{K}-40$ & 2020 & 0,654 & $0,647-0,718$ & $\mathrm{~Bq} / \mathrm{L}$ \\
\hline & $\mid-131$ & 2020 & $<0,289$ & --------- & $\mathrm{Bq} / \mathrm{L}$ \\
\hline & Cs-137 & 2020 & 0,032 & $0,029-0,074$ & $\mathrm{~Bq} / \mathrm{L}$ \\
\hline & Ra-226 & 2020 & 0,085 & $0,061-0,151$ & $\mathrm{~Bq} / \mathrm{L}$ \\
\hline \multirow{4}{*}{ Agua subterránea } & $\mathrm{K}-40$ & 2020 & 0,647 & $0,521-0,774$ & $\mathrm{~Bq} / \mathrm{L}$ \\
\hline & $\mathrm{I}-131$ & 2020 & $<0,108$ & ---------- & $\mathrm{Bq} / \mathrm{L}$ \\
\hline & Cs-137 & 2020 & $<0,026$ & ---------- & $\mathrm{Bq} / \mathrm{L}$ \\
\hline & Ra-226 & 2020 & 0,087 & $0,073-0,125$ & $\mathrm{~Bq} / \mathrm{L}$ \\
\hline
\end{tabular}




\section{Conclusiones}

El elaborado monitoreo de radiación a largo plazo y las observaciones radiológicas en el área de influencia del Centro Nuclear Oscar Miró Quesada de la Guerra "RACSO" permiten obtener información precisa sobre los niveles típicos y rangos de concentración de varios radionúclidos de importancia ambiental durante el período 19802020 permitiendo evidenciar que las actividades de las instalaciones se llevan de manera segura para el público y sin consecuencias radiosanitarias para el medio ambiente.

\section{Agradecimientos}

Se agradece a la Dirección de Servicios del IPEN por su apoyo para la ejecución del presente trabajo.

\section{Referencias}

[1] International Atomic Energy Agency. "Site Evaluation for Nuclear Installations". IAEA Safety Standards for protecting people and the environment. Specific Safety Requirements No. SSR-1. IAEA, Vienna, 2019.

[2] CS.Kim, SK. Lee, DM Lee, SW Choi. "Assessment of Environmental Radioactivity Surveillance Results around Korean Nuclear Power Utilization Facilities in 2017". Journal of Radiation Protection and Research, vol. 44, no. 3, pp. 118-126, 2019.
[3] H. Hinostroza, Y. Ravello, N. Cornejo, M. Mendoza, M. Montoya, A. Zuñiga, et al. "Neutrografía en el reactor peruano RP-10". Revista Peruana de Física, vol. 4, no. 3, pp. 1321, 1992.

[4] JM. Osores, S. Gonzáles, J. Martínez, E. López, R. Jara, A. Anaya. "Impacto radiológico ambiental del Centro Nuclear RACSO: programa de vigilancia radiológica ambiental". Informe Científico Tecnológico, vol. 2, pp. 205206, 2002.

[5] International Organization for Standardization (ISO), "Measurement of radioactivity, Gammaray emitting radionuclides, Generic test method using gamma-ray spectrometry". International Standard ISO 20042:2019(E).

[6] JM. Osores, P. Huamanlazo. Dosimetría ambiental en el Centro Nuclear Oscar Miró Quesada de la Guerra RACSO (1980-2015). Informe Científico Tecnológico, vol. 15, no. 1 pp. 31-34, 2015.

[7] International Commission on Radiological Protection. "Environmental Protection: Transfer Parameters for Reference Animals and Plants". ICRP Publication 114, Ann ICRP vol. 39, no. 6. 2009.

E-mails de todos los autores:

JM. Osores: ecorad1960@gmail.com

J. Martinez: imartinez@ipen.gob.pe

D. Huarachi: chuarachi@ipen.gob.pe

R. Koga: rkoga@ipen.gob.pe

R. Jara: riara@ipen.gob.pe

L. Huaman: Ihuaman@ipen.gob.pe 